\title{
FACTORIZABILITY OF MATRIX FUNCTIONS: A DIRECT PROOF
}

\author{
V. M. BABICH
}

\begin{abstract}
The factorization theorem mentioned in the title is about matrix-valued functions satisfying the Lipschitz condition on the real line and is related to the Riemann problem.
\end{abstract}

Many questions of mathematical physics reduce to what is called the Riemann problem, which consists in finding a certain scalar- or vector-valued piecewise holomorphic function. This function is assumed to be regular inside and outside of some contour, and its boundary values on that contour must exist and satisfy some special matching conditions. Solving a vector Riemann problem reduces to the factorization of matrix-valued functions. Provided the contour is finite, the proof of factorizability under natural assumptions dates back to Plemelj (1908); see [5]. A considerable contribution to this range of ideas was made in the books [6, 7]; among more recent publications we mention [8].

For many problems it is essential that the contour on which the matrix to be factorized is given coincide with the real axis. For an important and general class of such matrices, factorizability was proved by Gokhberg and Kreĭn in their classical paper [9. That proof is indirect. The factorization problem for an $(n \times n)$-matrix $E+G(t)$ ( $E$ is the unit matrix) is equivalent to finding linearly independent solutions of the homogeneous vector integral equation

$$
h(t)+\int_{0}^{+\infty} \tilde{G}(t-s) h(s) d s=0, \quad s, t \geq 0, \quad h \in \mathbb{C}^{n},
$$

where $\tilde{G}(t)$ is the matrix whose Fourier image coincides with $G(t)$. Integral equations similar to $(*)$ were the starting point of investigations in 9 . Besides the theory of integral equations, in the constructions of 9 an important part was played by Wiener's subtle results pertaining to harmonic analysis.

In the present paper, the author's aim is to prove the factorizability of matrix-valued functions of $t \in(-\infty,+\infty)$ along the lines suggested by Plemelj. This proof is direct. The matrices to be factored belong to the class $\operatorname{Lip} \alpha(0<\alpha<1)$, which is fairly standard in problems of mathematical physics. The fact that this class is natural was justified by many authors for finite contours; the same is done in the present paper for the contour $\mathbb{R}=(-\infty,+\infty)$. Professor A. P. Kachalov turned my attention to the fact that, with the help of an appropriate conformal transformation, the factorization problem in question can be reduced to the case of a finite contour. This makes it possible to use the existing results for finite contours and to simplify some constructions. However, we consider integral equations in the form in which they arise, with $\mathbb{R}^{1}$ in the role of the integration contour. It may be expected that these integral equations can be used for a numerical solution of the problem. Compared with the finite contour, the case of $\mathbb{R}^{1}$ has some specifics. A series of "additional theorems" have turned out to be necessary; hopefully, these results are of some interest in themselves.

2000 Mathematics Subject Classification. Primary 30E25.

Supported by RFBR (grant no. 08-01-00511). 


\section{SOME NOTATION}

1. $\mathbb{R}^{1}$ is the real axis: $\mathbb{R}^{1}=(-\infty,+\infty)$.

2. $\mathbb{C}$ is the complex plane.

3. $\operatorname{Lip} \alpha\left(\mathbb{R}^{1}\right)$ is the class of functions satisfying the Lipschitz condition on the closed line. For details and explanations concerning this and the following notation, see the Introduction.

4. $\operatorname{Lip} \alpha(D)$ is the class of functions defined on the set $D$ and satisfying the Lipschitz condition on $D$.

5. $\Pi_{+}$(respectively, $\left.\Pi_{-}\right)$is the closed half-plane $\{z: z \in \mathbb{C}, \operatorname{Im} z \geq 0\}$ (respectively, $\{z: z \in \mathbb{C}, \operatorname{Im} z \leq 0\}$ ), with the point at infinity adjoined.

6. $E$ is the unit matrix.

7. $\mathbb{Z}$ is the set of integers.

8. If $\psi=\left(\psi_{1}, \psi_{2}, \ldots, \psi_{n}\right)$ is a vector, then $|\psi|:=\sqrt{\sum_{j=1}^{n}\left|\psi_{j}\right|^{2}}$.

9. If $L=\left(L_{i j}\right)$ is an $(n \times n)$-matrix, then $\|L\|:=\sqrt{\sum_{i, j=1}^{n}\left|L_{i j}\right|^{2}}$.

10. $\square$ marks the end of a proof.

\section{$\S 1$. INTRODUCTION}

Here we consider the factorization problem for $(n \times n)$-matrices $M(t)$ the entries of which are functions of a parameter $t \in \mathbb{R}^{1}$. For a theoretical problem of mathematical physics, success in its solution is often related with an adequate choice of the function spaces where the corresponding constructions are performed. There is a good reason to say that one of such spaces is represented by the Lipschitz classes 1 with exponent $\alpha$ $(0<\alpha<1)$, and also the classes of vectors and matrices with Lipschitz components. We turn to the definitions. We write $f(t) \in \operatorname{Lip} \alpha\left(\mathbb{R}^{1}\right)$, and say that $f(t)$ is a Lipschitz function on the closed line 2 The same definition of the Lipschitz property on the closed line was used by Gakhov (see, e.g., [10, Chapter 1, §4]) if on any segment $|t| \leq B$ we have

$$
\left|f\left(t^{\prime}\right)-f\left(t^{\prime \prime}\right)\right| \leq \text { const }\left|t^{\prime}-t^{\prime \prime}\right|^{\alpha}, \quad 0<\alpha<1, \quad\left|t^{\prime}\right|,\left|t^{\prime \prime}\right| \leq B
$$

Here const does not depend on $t^{\prime}, t^{\prime \prime}$. For $|t| \geq B_{1}=$ const $>0$, we require that

$$
\left|f\left(t^{\prime}\right)-f\left(t^{\prime \prime}\right)\right| \leq \mathrm{const}\left|\frac{1}{t^{\prime}}-\frac{1}{t^{\prime \prime}}\right|^{\alpha}, \quad 0<\alpha<1, \quad\left|t^{\prime}\right|,\left|t^{\prime \prime}\right| \geq B_{1}
$$

( $B$ and $B_{1}$ may be arbitrary positive constants).

Let $D$ be the segment $|t| \leq B$ or the exterior of the interval $(-B,+B)$, i.e., $D=$ $\{t:|t| \geq B\}$. Then the relation $f \in \operatorname{Lip} \alpha(D)$ means (1.1) in the first case and (1.2) in the second. We shall also need Lipschitz functions $f(t)$ defined for complex $t$. Let $D$ be the closure of some domain lying in $\mathbb{C}$. If $D$ is compact, $f \in \operatorname{Lip} \alpha(D)$ means that (1.1) is fulfilled for $t^{\prime}, t^{\prime \prime} \in D$. If $D$ is unbounded, then $f \in \operatorname{Lip} \alpha(D)$ means that for any $B, B_{1}=$ const $>0$ we have $f \in \operatorname{Lip} \alpha(D \cap\{t:|t| \leq B\})$ and $f \in \operatorname{Lip} \alpha\left(D \cap\left\{t:|t| \geq B_{1}\right\}\right)$, i.e., (1.2) is fulfilled.

If the components of a vector $\varphi$ or a matrix $M$ belong to $\operatorname{Lip} \alpha(D)$, then we say that $\varphi$ or $M$ are Lipschitz on $D$, and write $\varphi \in \operatorname{Lip} \alpha(D))$ or $M \in \operatorname{Lip} \alpha(D)$. Obviously, the matrices that are Lipschitz on $D$ form a ring, and on this ring we can introduce a norm.

\footnotetext{
${ }^{1}$ We talk of the Lipschitz rather than Hölder classes, because our classes Lip $\alpha$ are always onedimensional. Precisely these classes were introduced by Lipschitz in 1864. Later, Hölder extended the notion of "being Lipschitz" to the many-dimensional case.

${ }^{2}$ The term "closed line" is borrowed from 9 .
} 
Relation (1.2) implies that, for real $t$, the function $f(t)$ has limits as $t \rightarrow+\infty$ and as $t \rightarrow-\infty$, and that these limits coincide and $f(t)-f(\infty)=O\left(|t|^{-\alpha}\right)$. If $D=\Pi_{ \pm}$or $D=\Pi_{ \pm} \cap\{|t| \geq B\}$, then the limit of $f(t)$ at infinity exists as well, and

$$
f(t)=f(\infty)+O\left(|t|^{-\alpha}\right)
$$

where the remainder term $O\left(|t|^{-\alpha}\right)$ admits an estimate uniform in $\arg t$.

With obvious modifications, the above properties of Lipschitz functions carry over to the Lipschitz vectors and matrices.

Now, we state the main result of the paper. Let $\mathcal{F}$ be the class of matrices $M(t) \in$ $\operatorname{Lip} \alpha\left(\mathbb{R}^{1}\right)$ such that $\operatorname{det} M(t) \neq 0$; in particular, $\operatorname{det} M(\infty) \neq 0$. The main result of the paper is the proof of the fact that any matrix in $\mathcal{F}$ is factorizable. More precisely, the following statement is true.

Theorem 1.1. Any $M \in \mathcal{F}$ admits a representation in the form ${ }^{3}$

$$
M=K_{+}(t) \Xi K_{-}(t) .
$$

Here $K_{ \pm} \in \mathcal{F}$ and

$$
\Xi=\left(\begin{array}{ccc}
\zeta^{\varkappa_{1}} & & \\
& \ddots & \\
& & \zeta^{\varkappa_{n}}
\end{array}\right) ; \quad \varkappa_{j} \in \mathbb{Z}, \quad \zeta=\frac{t-i}{t+i} .
$$

The matrix $K_{+}$(respectively, $\left.K_{-}\right)$extends analytically to the half-plane $\Pi_{+}$(respectively, $\left.\Pi_{-}\right)$, and for the corresponding extensions we have $K_{+} \in \operatorname{Lip} \alpha\left(\Pi_{+}\right)$and $\operatorname{det} K_{+}(z) \neq 0$, in particular, $\operatorname{det} K_{+}(\infty) \neq 0$ (respectively, $K_{-} \in \operatorname{Lip} \alpha\left(\Pi_{-}\right)$and $\operatorname{det} K_{-}(z) \neq 0$, in particular, $\left.\operatorname{det} K_{-}(\infty) \neq 0\right)$.

The representation (1.3), (1.4) is called the left factorization of the matrix $M(t)$. The existence of the right factorization is proved similarly:

$$
M=K_{-}^{r}(t) \Xi^{r} K_{+}^{r}(t) .
$$

Here $\Xi^{r}$ has the form (1.4) with, in general, some other $\varkappa_{s}$ and $K_{ \pm}^{r} \in \mathcal{F}$.

Formulas (1.3) and (1.4) serve as a starting point for the proof of the following important statement.

Theorem 1.2 (Muskhelishvili).

$$
\sum_{i=1}^{n} \varkappa_{i}=\frac{1}{2 \pi} \int_{-\infty}^{+\infty} d \arg \operatorname{det} M(t):=\operatorname{ind} M .
$$

In the case of a right factorization, for the sum of the indices we have the same Muskhelishvili formula:

$$
\sum_{i=1}^{n} \varkappa_{i}^{r}=\frac{1}{2 \pi} \int_{-\infty}^{+\infty} d \arg \operatorname{det} M(t):=\operatorname{ind} M .
$$

It is of interest to note that, for $n=1$, in the scalar (rather than matrix) case, it is known that the factorization of $M(t) \in \mathcal{F}$ can be found explicitly. These explicit expressions lead to a formula of the form (1.3), where the $K_{ \pm}$belong to the same function classes as indicated in Theorem 1.1.

In what follows, it is assumed throughout that $0<\alpha<1$. The case of $\alpha=1$ is specific: almost all statements and proofs require modification in this case. In order not to overload our presentation, we restrict ourselves to the case of $0<\alpha<1$.

${ }^{3} \mathrm{~A}$ representation as in (1.3) or (1.5) can be found in $[9$, but with other function spaces. 


\section{§2. Deduction of The BASIC Integral EQUATION}

First, we consider the relationship between the homogeneous Riemann problem and factorization.

By the homogeneous Riemann problem on $\mathbb{R}^{1}$ we mean the problem of finding a piecewise holomorphic vector $\varphi(z)$,

$$
\varphi(z):=\varphi_{+}(z) \quad \text { for } \quad z \in \Pi_{+}, \quad \varphi(z):=\varphi_{-}(z) \text { for } z \in \Pi_{-},
$$

such that in the half-planes $\Pi_{ \pm}$the vector-valued functions are regular 4 Moreover, it is assumed that the functions $\varphi_{ \pm}(z)$ have limit values on the real axis $\mathbb{R}^{1}$ and that these limit values satisfy the linear relation

$$
\varphi_{+}(t)=M(t) \varphi_{-}(t),
$$

where $M(t)$ is a nonsingular matrix whose entries are functions of $t \in \mathbb{R}^{1}$. If problem (2.1) has $n$ linearly independent solutions, then we can construct matrices $\Phi_{+}$and $\Phi_{-}$the columns of which are composed of the components of the solutions $\varphi_{ \pm}$of the Riemann problem. Obviously,

$$
\Phi_{+}(t)=M(t) \Phi_{-}(t) .
$$

Since the columns of the matrix $\Phi_{-}(t)$ are linearly independent, this matrix is invertible, and we can rewrite $(2.2)$ in the form

$$
M(t)=\Phi_{+}(t)\left(\Phi_{-}(t)\right)^{-1},
$$

arriving at a factorization of the matrix $M(t)$.

Of course, the formal arguments outlined here need specification, which will be done later, but already now we can conclude that the factorization problem reduces to the study of linearly independent solutions of the Riemann problem (2.1).

It turns out that the general plan of the investigation of problem (2.1), which was suggested long ago by Plemelj for the case of a finite contour, works also in our case, where the contour coincides with the real line, but the unboundedness of the contour complicates the study greatly at all steps.

Now we make the setting of problem (2.1) more precise. In what follows, it is assumed throughout that $M \in \mathcal{F}$ and $M(\infty)=E$. As to $\varphi(z)$, we assume that the functions $\varphi(z)$ are regular in $\Pi_{ \pm}$, the limit values as $\operatorname{Im} z \rightarrow \pm 0$ exist and belong to $\operatorname{Lip} \alpha\left(\mathbb{R}^{1}\right)$, and

$$
\varphi_{ \pm}(z)=\gamma+O\left(|z|^{-\alpha}\right), \quad z \rightarrow \infty
$$

Here $\gamma$ is a constant vector $\left(\gamma\right.$ is the same for $\Pi_{+}$and for $\Pi_{-}$, which agrees with the fact that $M(\infty)=E)$. The estimate $O\left(|z|^{-\alpha}\right)$ is uniform in $\arg z$.

Equation (2.1) shows that, in order to find $\varphi_{+}(t)$, it suffices to determine $\varphi_{-}(t)$; this allows us to deduce the equation only for $\varphi_{-}(t)$. In essence, the corresponding arguments repeat, for the case of $\mathbb{R}^{1}$, the deduction of a similar equation in the case of a finite contour (see also [1]).

The condition that $\varphi_{-}(t) \in \operatorname{Lip} \alpha\left(\mathbb{R}^{1}\right)$ is the boundary value on $\mathbb{R}^{1}$ of a vector holomorphic in $\Pi_{-}$and equal to $\gamma$ at infinity (see (2.4)), is equivalent (see ([7, Chapter 1 , $\S 4])$ to the relation

$$
\frac{1}{2}\left(\varphi_{-}(t)-\gamma\right)=\frac{1}{2 \pi i} \int_{\mathbb{R}^{1}} \frac{\left(\varphi_{-}(s)-\gamma\right)}{s-t} d s, \quad t \in \mathbb{R}^{1}
$$

\footnotetext{
${ }^{4} \mathrm{~A}$ vector $\varphi(z)=\left(\varphi_{1}(z), \ldots, \varphi_{n}(z)\right)$ is said to be regular $(\Longleftrightarrow$ holomorphic) in a domain if all its components are regular ( $\Longleftrightarrow$ holomorphic) in this domain.
} 
Identity (2.5) is a consequence of the Cauchy integral formula and the Sokhotskiu formulas for the limit values of a Cauchy type integral. The integral in (2.5) is understood in the principal value sense.

A similar condition for $\varphi_{+}(t)=M(t) \varphi_{-}(t)$ has the form

$$
\frac{1}{2}\left(M(t) \varphi_{-}(t)-\gamma\right)=\frac{1}{2 \pi i} \int_{\mathbb{R}^{1}} \frac{\left(M(s) \varphi_{-}(s)-\gamma\right)}{s-t} d s, \quad t \in \mathbb{R}^{1} .
$$

We denote $\varphi_{-}(z)-\gamma:=\varphi_{-}^{\circ}(z)$. Identities (2.5) and (2.6) take the form

$$
\begin{aligned}
\frac{1}{2} \varphi_{-}^{\circ}(t) & =-\frac{1}{2 \pi i} \int_{\mathbb{R}^{1}} \frac{\varphi_{-}^{\circ}(s)}{s-t} d s, \quad t \in \mathbb{R}^{1}, \\
\frac{1}{2} M(t) \varphi_{-}^{\circ}(t) & =\frac{1}{2 \pi i} \int_{\mathbb{R}^{1}} \frac{M(s) \varphi_{-}^{\circ}(s)}{s-t} d s+f(t), \quad t \in \mathbb{R}^{1},
\end{aligned}
$$

where

$$
f(t)=-\frac{1}{2}(M(t) \gamma-\gamma)+\frac{1}{2 \pi i} \int_{\mathbb{R}^{1}} \frac{(M(s) \gamma-\gamma)}{s-t} d s .
$$

We apply the matrix $(M(t))^{-1}$ to both sides of $(2.8)$ and add the resulting identity to (2.7). As a result, we obtain the desired integral equation for $\varphi^{\circ}$ :

$$
\varphi^{\circ}(t)=\frac{1}{2 \pi i} \int_{\mathbb{R}^{1}} \frac{M(t)^{-1} M(s)-E}{s-t} \varphi^{\circ}(s) d s+M(t)^{-1} f(t) .
$$

If the matrix $M(t)$ satisfies the conditions imposed in the Introduction, then equation (2.10) turns out to be Fredholm.

The formulations and proofs of the theorems that follow are to a great extent influenced by the fact that we deal with an infinite contour.

Theorem 2.1. Let $m(t)$ be an $(n \times n)$-matrix belonging to the class $\mathcal{F}$, and let

$$
K(t, s)=\frac{1}{2 \pi i} \frac{m(t)^{-1} m(s)-E}{t-s} .
$$

Then the integral operator

$$
K \varphi:=\int_{\mathbb{R}^{1}} K(t, s) \varphi(s) d s
$$

is compact on any $\mathrm{L}_{p}\left(\mathbb{R}^{1}\right), p>1$.

(All Fredholm integral operators occurring in the paper have kernels of the form (2.11); by the norm of a vector $\varphi(t)=\left\{\varphi_{1}(t), \ldots, \varphi_{n}(t)\right\}$ one can mean $\sum_{j=1}^{n}\left\|\varphi_{j}(t)\right\|_{\mathrm{L}_{p}}$, or $\||\varphi(t)|\|_{L_{p}}$, where $|\varphi(t)|=\sqrt{\sum_{j=1}^{n}\left|\varphi_{j}(t)\right|^{2}}$.)

Theorem 2.2. The free term in (2.10) belongs to $\operatorname{Lip} \alpha\left(\mathbb{R}^{1}\right), 0<\alpha<1$, and is of order of $O\left(|t|^{-\alpha}\right)$ as $t \rightarrow \infty$.

Theorem 2.3. Suppose that

$$
\Psi(t)=\int_{\mathbb{R}^{1}} K(t, s) \Psi(s) d s+F(t)
$$

where $K(t, s)$ is a kernel of the form (2.11), $F(t) \in \operatorname{Lip} \alpha\left(\mathbb{R}^{1}\right), 0<\alpha<1, F(\infty)=0$, and $\Psi(t) \in \mathrm{L}_{p}$, where $p$ is such that $F(t) \in \mathrm{L}_{p}\left(\mathbb{R}^{1}\right)$ (for this it suffices to assume that $\alpha p>1)$. Then $\Psi(t) \in \operatorname{Lip} \alpha\left(\mathbb{R}^{1}\right)$, and as $t \rightarrow \infty$ we have $\Psi(t)=O\left(|t|^{-\alpha}\right)$. 
Theorem 2.4. If

$$
\Psi(t)=\int_{\mathbb{R}^{1}} K(t, s) \Psi(s) d s,
$$

where $K(t, s)$ is a kernel of the form (2.11), and $\Psi(t) \in \mathrm{L}_{p}$, where $p$ is an arbitrary number exceeding 1 , then $\Psi(t)=\frac{1}{t} \Psi_{1}(t)$, where $\Psi_{1}$ is a function Lipschitz at infinity: $\Psi_{1}(t) \in \operatorname{Lip} \alpha\{t:|t| \geq$ const $>0\}$.

We shall also need the following statement.

Theorem 2.5. Let $\omega(t) \in \operatorname{Lip} \alpha(\mathbb{R})$, let $\omega(\infty)=0$, and let $f(z)$ be the Cauchy integral

$$
f(z)=\frac{1}{2 \pi i} \int_{\mathbb{R}^{1}} \frac{\omega(s) d s}{s-z} .
$$

Then $f(z) \in \operatorname{Lip} \alpha\left(\Pi_{ \pm}\right)$and $f(\infty)=0$. If, moreover, for $|t| \geq B=$ const $>0$ we have $\omega(t)=\frac{\omega_{1}(t)}{t}$ with $\omega_{1}(t) \in \operatorname{Lip} \alpha(|t| \geq B)$, then for $|z| \geq B$ we have $f(z)=\frac{1}{z} f_{1}(z)$ with $f_{1}(z) \in \operatorname{Lip} \alpha\left(\Pi_{ \pm} \cap|z| \geq B\right)$.

Proof. Observe that the components of the vector $f(t)=M(t) \gamma-\gamma$ belong to $\operatorname{Lip} \alpha\left(\mathbb{R}^{1}\right)$, $0<\alpha<1$, and vanish at infinity. In [9, Chapter 1] it is proved that the Cauchy integral with such a density belongs to $\operatorname{Lip} \alpha\left(\mathbb{R}^{1}\right)$ and admits an estimate of order $O\left(|t|^{-\alpha}\right)$ at infinity, and Theorem 2.5 follows.

Theorems 2.1-2.4 will be proved in the Appendices. Largely, the proofs are based on estimates close to those in [6, Chapter 2], [10, Chapter 1], and [11, Chapter 1].

\section{§3. Allied problem And aCCOMPanying PRoblem}

Following the way suggested by Plemelj, we put the question as to whether the function $\varphi(t)=\varphi^{\circ}(t)+\gamma$, where $\varphi^{\circ}(t)$ is the solution of the integral equation (2.10), solves the Riemann problem (1.2). First, we note that $\varphi_{-}^{\circ}(t) \in \mathrm{L}_{p}$ for some $p>1$. Also, for $f(t) \not \equiv 0$, we require that $p \alpha>1$; then the free term of the equation belongs to the same space $\mathrm{L}_{p}$ as $\varphi_{-}^{\circ}(t)$, and, by Theorems 2.2 and $2.3, \varphi_{-}^{\circ}(t)$ belongs to $\operatorname{Lip} \alpha\left(\mathbb{R}^{1}\right)$ and admits estimates of order $O\left(|t|^{-\alpha}\right)$ as $t \rightarrow \infty$.

We introduce the following piecewise holomorphic vector: $\psi(z)=\psi_{+}(z)$ for $z \in \Pi_{+}$ and $\psi(z)=\psi_{-}(z)$ for $z \in \Pi_{-}$, where

$$
\psi_{+}(z):=\frac{1}{2 \pi i} \int_{\mathbb{R}^{1}} \frac{\varphi_{-}^{\circ}(s)}{s-t} d s, \quad z \in \Pi_{+},
$$

and

$$
\psi_{-}(z):=\frac{1}{2 \pi i} \int_{\mathbb{R}^{1}} \frac{M(s) \varphi_{-}^{\circ}(s)}{s-t} d s+\frac{1}{2 \pi i} \int_{\mathbb{R}^{1}} \frac{M(s) \gamma-\gamma}{s-z} d s, \quad z \in \Pi_{-} .
$$

Formulas (2.7) and (2.8) are equivalent (respectively) to the relations $\psi_{+}(t)=0$ and $\psi_{-}(t)=0$, and $\psi=O\left(|z|^{-\alpha}\right)$ as $z \rightarrow \infty$, i.e., $\varphi_{-}$solves the Riemann problem (2.1) if and only if $\psi(z) \equiv 0$. Suppose we know only that $\varphi_{-}^{\circ}(t) \varphi_{-}^{\circ}(t)$ belongs to $L_{p}, p>1$, and solves equation (2.10). This equation is equivalent to the relation

$$
\psi_{+}(t)=M(t)^{-1} \psi_{-}(t) .
$$

The problem of finding $\psi_{ \pm}(t)$ satisfying (3.3) will be called the problem accompanying problem (1.2) (see [7, Chapter 1]).

The above considerations lead to the following statement. 
Theorem 3.1. Suppose that problem (3.3) has only the trivial solution in the class of functions $\psi \in \operatorname{Lip} \alpha\left(\mathbb{R}^{1}\right), 0<\alpha<1$, with $\psi_{ \pm}(t) \rightarrow 0$ as $t \rightarrow \infty$. Then any solution $\varphi_{-}^{\circ} \in \mathrm{L}_{p}\left(\mathbb{R}^{1}\right)$ (with $p \alpha>1$ for $f(t) \neq 0$ ) of the integral equation (2.10) gives rise to a solution $\varphi_{-}=\varphi_{-}^{\circ}+\gamma$ of the Riemann problem (2.1).

The next question is the solvability of the integral equation (2.10) when $\gamma=$ const. As in the case of a finite contour, we consider the adjoint equation. Here, it will be an equation in the space of functionals over $\mathrm{L}_{p}\left(\mathbb{R}^{1}\right)$, i.e., in the space $\mathrm{L}_{p^{\prime}}\left(\mathbb{R}^{1}\right)$, where $\frac{1}{p^{\prime}}+\frac{1}{p}=1$. The corresponding adjoint equation is the homogeneous Fredholm equation

$$
\psi_{+}^{\prime}(t)=\frac{1}{2 \pi i} \int_{\mathbb{R}^{1}} \frac{M(t)^{T}\left(M(s)^{T}\right)^{-1}-E}{s-t} \psi_{+}^{\prime}(s) d s,
$$

where $T$ denotes transposition; when possible, our notation is as in [7].

Consider the Riemann problem "allied" to problem (1.2) (see 7, Chapter 1]):

$$
\varphi_{+}^{\prime}(t)=\left(M(t)^{T}\right)^{-1} \varphi_{-}^{\prime}(t)
$$

with the matrix $\left(M^{T}\right)^{-1}$ contragredient to the matrix $M$; then the problem accompanying (3.5) has the form

$$
\psi_{+}^{\prime}(t)=M(t)^{T} \psi_{-}^{\prime}(t) .
$$

The integral equation corresponding to problem (3.6) coincides with (3.4).

Now we prove the following important statement.

Theorem 3.2. Suppose that there exists $0<\beta<1$ such that problem (3.6) admits only the trivial solution in the class of functions belonging to $\operatorname{Lip} \beta\left(\Pi_{ \pm}\right)$and tending to zero at infinity (and thus, dominated at infinity by $\frac{\text { const }}{|z|^{\beta}}$ unformly in $\arg z$ ). Then equation (2.10) is solvable for any vector $\gamma$.

Proof. By Theorem 3.1, which is applicable in the case under consideration, the solutions of equation (3.4) extend analytically to the upper half-plane. At infinity, the $\psi_{ \pm}^{\prime}(t)$ will be of order of $\frac{1}{t}$ (see Theorem 2.4). For an arbitrary solution $\psi_{ \pm}^{\prime}(t)$ of the homogeneous equation (3.4), the integral

$$
\int_{\mathbb{R}^{1}} M^{-1} f_{\gamma}(t) \psi_{ \pm}^{\prime}(t) d t
$$

where $f_{\gamma}(t)$ is as in (2.9), converges, because the $\psi_{ \pm}^{\prime}(t)$ are of order $\frac{1}{t}$ as $t \rightarrow \infty$, while $f_{\gamma}(t)=O\left(|t|^{-\alpha}\right)$ and $M^{-1}$ tends to $E$ at infinity.

The integral (3.7) can be rewritten as follows:

$$
\int_{\mathbb{R}^{1}} f_{\gamma}(t)\left(M^{-1}\right)^{T} \psi_{+}^{\prime}(t) d t=\int_{\mathbb{R}^{1}} f_{\gamma}(t) \psi_{-}^{\prime}(t) d t .
$$

The integral on the right is equal to zero, because $\psi_{-}^{\prime}(t)$ extends analytically to $\Pi_{-}$and is of order $O\left(\frac{1}{z}\right)$ at infinity, and the function $f_{\gamma}(t)$ also admits extension and is of order $O\left(|z|^{-\alpha}\right)$ at infinity.

If the conditions of Theorems 3.1 and 3.2 are fulfilled, then, as in [7, we let the vector $\gamma$ take the values

$$
e_{1}=(1,0, \ldots, 0), e_{2}=(0,1, \ldots, 0), \ldots, e_{n}=(0,0, \ldots, 1) .
$$

The general solution of the homogeneous Riemann problem has the form

$$
\varphi(z)=\delta_{1} \varphi^{1}+\cdots+\delta_{n} \varphi^{n}+\delta_{n+1} \varphi^{n+1}+\cdots+\delta_{m} \varphi^{m} .
$$


Here the $\delta_{j}$ are arbitrary coefficients, and $m<\infty$, because the $\varphi^{j}, j>n$, correspond to solutions of a homogeneous Fredholm equation, and the number of such linearly independent solutions is finite.

\section{§4. Generalization of formula (3.9)}

In this section, our aim is to generalize formula (3.9) to cover the case where $\varphi(z)$ can have a pole at the point $-i$. We start with a lemma.

Lemma. There exists $s$ such that any root of a solution of (2.1) belonging to $\operatorname{Lip} \alpha\left(\Pi_{ \pm}\right)$, $0<\alpha<1$, has order at most $s$. The order of the root at infinity also does not exceed $s$.

The proof does not differ from the case of a finite contour (see [7]).

Let $\varphi(z)$ be a solution of the Riemann problem (2.1) that belongs to $\operatorname{Lip} \alpha\left(\Pi_{+}\right)$, $0<\alpha<1$, and is Lipschitz in $\Pi_{-}$with the point $-i$ removed. The latter means that, for any neighborhood $\sigma$ of the point $-i$, the function $\varphi(z)$ belongs to $\operatorname{Lip} \alpha(\Pi \backslash \sigma), 0<\alpha<1$. Next, assume that the order 5 at the point $-i$ of the function $\varphi(z)$ is at least $-d(d$ is a "large" number). Thus, $\varphi(z)$ can have a pole of order not exceeding $d$,

$$
\varphi(z)=O\left(\frac{1}{|z+i|^{d}}\right) .
$$

We introduce the piecewise holomorphic vector

$$
\varphi_{+}^{*}(z)=\varphi_{+}(z), \quad z \in \Pi_{+}, \quad \varphi_{-}^{*}(z)=\varphi_{-}(z)\left(\frac{z+i}{z-i}\right)^{d}, z \in \Pi_{-} .
$$

This vector solves problem (2.1) with the matrix $M(t)$ replaced by $M(t)\left(\frac{t-i}{t+i}\right)^{d}$ :

$$
\varphi_{+}^{*}(t)=M(t)\left(\frac{t-i}{t+i}\right)^{d} \varphi_{-}^{*}(t) .
$$

If $\varphi^{*}(z) \in \operatorname{Lip} \alpha\left(\Pi_{ \pm}\right), 0<\alpha<1$, is a solution of (4.2), then the corresponding vector $\varphi(z)$ is a solution of (2.1) such that it belongs to $\operatorname{Lip} \alpha\left(\Pi_{-}\right)$, is Lipschitz in $\Pi_{-}$with the point $-i$ removed, and has order $r \geq-d$ at this point.

If $d$ is sufficiently large, then the Riemann problem (4.2) satisfies the conditions of both Theorems 3.1 and 3.2. Indeed, the problem accompanying (4.2) has the form

$$
\psi_{+}^{*}(t)=M(t)^{-1}\left(\frac{t+i}{t-i}\right)^{d} \psi_{-}^{*}(t) .
$$

Should this problem have a solution in $\operatorname{Lip} \alpha\left(\Pi_{ \pm}\right)$, the solution $\psi_{+}(t)=M(t)^{-1} \psi_{-}(t)$ of the problem accompanying (2.1) would have a root of order at least $d$, which is impossible for sufficiently large $d$ and $\psi \not \equiv 0$. Similarly, for sufficiently large $d$ the solution of class $\operatorname{Lip} \alpha\left(\Pi_{ \pm}\right)$of the problem

$$
\varphi_{+}^{* \prime}(t)=\left(M(t)^{T}\right)^{-1}\left(\frac{t+i}{t-i}\right)^{d} \varphi_{-}^{* \prime}(t)
$$

which is allied to (4.2), would give rise to a solution of problem (3.5) that has a zero of order at least $d$ at the point $-i$. For sufficiently large $d$ this is impossible if $\varphi_{-}^{* \prime}(t) \not \equiv 0$. Thus, the integral equation of the form (2.10) that corresponds to (4.2) is solvable for any vector $\gamma=$ const, and the solution of it leads to a solution of (4.2). We arrive at the desired generalization of formula (3.9): all solutions $\varphi(z)$ of the Riemann problem (2.1) such that $\varphi_{+} \in \operatorname{Lip} \alpha\left(\Pi_{+}\right), \varphi_{-} \in \operatorname{Lip} \alpha\left(\Pi_{-} \backslash(-i)\right)$, and the order of $\varphi_{-}$at the point $-i$ is at least $d$, are representable in the form (3.9):

$$
\varphi(z)=\delta_{1} \varphi^{1}+\cdots+\delta_{n} \varphi^{n}+\delta_{n+1} \varphi^{n+1}+\cdots+\delta_{m} \varphi^{m} .
$$

\footnotetext{
${ }^{5}$ Following the existing tradition, we say that $\varphi(z)$ has order $r \in \mathbb{Z}$ at a point $z_{0}$ if the ratio $\frac{\varphi(z)}{\left(z-z_{0}\right)^{r}}=$ $\psi(z)$ is regular at $z_{0}$ and $\psi\left(z_{0}\right) \neq 0$.
} 
Here the $\delta_{j}$ are arbitrary coefficients, $m=m(d)<\infty, \varphi^{j}(z) \underset{z \rightarrow \infty}{\longrightarrow} e_{j}$ if $j \leq n$ and $\varphi^{j}(z) \underset{z \rightarrow \infty}{\longrightarrow} 0$ if $j>n$, and $e_{1}=(1,0, \ldots, 0), \ldots, e_{n}=(0,0, \ldots, 1)$. Formula (4.4) will serve as a starting point for the construction of a canonical system of solutions of the Riemann problem (2.1). Precisely this system of $n$ solutions entails the desired factorization of the matrix $M(t)$.

\section{§5. Canonical system of solutions of the Riemann problem}

Now everything is ready for the proof of the existence of a canonical system of solutions, in accordance with Plemelj's plan. The plan is reproduced almost exactly, but the role of the point $z=\infty$ now is played by the point $-i$.

We pass to constructing a canonical system. Suppose that a nonzero vector $\chi^{1}(z)$ belongs to $\operatorname{Lip} \alpha\left(\Pi_{+}\right)$and is Lipschits in $\Pi_{-}$with the point $-i$ removed, and assume that the order of $\chi^{1}(z)$ is the maximal possible. Since the possible orders are bounded from above, such a vector $\chi^{1}(z)$ exists.

We shall construct a canonical system of solutions Lipschitz in $\Pi_{ \pm}$(without the point $-i$ ) by induction. We already have the vector $\chi^{1}(z)$. Suppose that $\chi^{1}(z), \chi^{2}(z), \ldots, \chi^{k}(z)$ have already been constructed. For the role of $\chi^{k+1}(z)$ we take a vector of maximal order at the point $-i$ among all solutions of the form (4.4) not expressible linearly in terms of $\chi^{1}(z), \chi^{2}(z), \ldots, \chi^{k}(z)$ by a formula of type

$$
\varphi(z)=\sum_{j=1}^{k} p_{j}\left(\frac{1}{z+i}\right) \chi^{j}(z),
$$

where $p_{j}\left(\frac{1}{z+i}\right)$ is a polynomial in $\frac{1}{z+i}$.

We show that such a $\chi^{k+1}(z)$ exists indeed. First, observe that the determinant

$$
\Delta(z):=\operatorname{det}\left(\varphi^{1}, \varphi^{2}, \ldots, \varphi^{n}\right),
$$

the columns of which are the components of the vectors $\varphi^{j}(z)$ (see formula (4.4)), tends to 1 as $z \rightarrow \infty$, and thus, is not identically zero. Suppose that all solutions of problem (2.1) in the class in question can be expressed linearly in terms of $\chi^{1}(z), \chi^{2}(z), \ldots, \chi^{k}(z)$; then the same is true for the vectors $\varphi^{1}, \varphi^{2}, \ldots, \varphi^{n}$. Then for each vector $\varphi^{j}$ we have

$$
\varphi^{l}(z)=\sum_{j=1}^{k} p_{j}^{l}\left(\frac{1}{z+i}\right) \chi^{j}(z) .
$$

We substitute (5.3) in (5.2). The determinant $\Delta$ can be represented as a linear combination of determinants of the form

$$
\operatorname{det}\left(\chi^{j_{1}}, \chi^{j_{2}}, \ldots, \chi^{j_{n}}\right)
$$

$\left(j_{1}, \ldots, j_{n}\right.$ take values from 1 to $\left.k\right)$ with polynomial coefficients. All determinants (5.4) are equal to zero, because any collection of length $n$ formed by $k<n$ numbers must have repetitions, i.e., each determinant (5.4) must have at least two equal columns. We arrive at a contradiction, and this shows that the collection of vectors $\left(\chi^{1}, \chi^{2}, \ldots, \chi^{n}\right)$ can be constructed. This collection is the desired canonical system.

It is important to note that none of the vectors $\chi^{j}$ can have a zero at a point $c \in \Pi_{ \pm}$, $c \neq-i$. Indeed, if $c \notin \mathbb{R}^{1}$, then, multiplying $\chi^{j}$ by $\frac{z+i}{z-c}$, we see that the order of $\chi^{j}$ at the point $-i$ is not maximal. The case of $c \in \mathbb{R}^{1}$ is more difficult and can be settled by the methods of [7]. So, $\chi^{j}(z) \neq 0$ for $z \in \Pi_{+} \cup \Pi_{-} \backslash(-i)$. At infinity, $\chi^{j}(z)$ has a finite limit distinct from zero. Denote by $-\varkappa_{j}$ the order of $\chi^{j}$ at the point $-i$; then

$$
\chi^{j}=\tilde{\chi}^{j}\left(\frac{z+i}{z-i}\right)^{-\varkappa_{j}}, \quad \tilde{\chi}^{j} \in \operatorname{Lip} \alpha\left(\Pi_{ \pm}\right) .
$$


Put

$$
\begin{aligned}
& \chi^{j}(z)=\chi_{-}^{j}(z), \quad z \in \Pi_{-}, \\
& \chi^{j}(z)=\chi_{+}^{j}(z), \quad z \in \Pi_{+} .
\end{aligned}
$$

Let $\Phi_{+}$(respectively, $\Phi_{-}$) be the matrix the columns of which are the vectors $\chi_{+}^{j}$ (respectively, $\chi_{-}^{j}$ ). Obviously, on the real line we have

$$
M \Phi_{-}=\Phi_{+}
$$

or

$$
M \tilde{\Phi}_{-}\left(\begin{array}{lll}
\zeta^{-\varkappa_{1}} & & \\
& \ddots & \\
& & \zeta^{-\varkappa_{n}}
\end{array}\right)=\Phi_{+} .
$$

The columns of the matrix $M \tilde{\Phi}_{-}(z)$ are the vectors $\tilde{\chi}^{j}(z), z \in \Pi_{-}$(see (5.5)). It is not hard to check (see [7]) that $\operatorname{det} \tilde{\Phi}_{-} \neq 0$ and $\operatorname{det} \Phi_{+} \neq 0$ (in particular, at $z=\infty$ ). Then (5.9) implies immediately that

$$
M(t)=\Phi_{+} \Xi\left(\tilde{\Phi}_{-}\right)^{-1} .
$$

Formula (5.10) differs from (1.3) only in notation. This completes the proof of Theorem 1.1.

\section{APPEndix 1}

The proof of Theorem 2.1 is based on the well-known statement that the norm limit of compact operators is a compact operator.

$1^{\circ}$. In this connection, we introduce the function

$$
\eta_{A}(s)= \begin{cases}1, & |s| \leq A, \\ 0, & |s|>A,\end{cases}
$$

where $A$ is a "large" positive number. The kernel

$$
K(t, s)=\frac{m^{-1}(t) m(s)-E}{s-t}
$$

of the integral operator (2.12) splits into the sum of four terms:

$$
\begin{aligned}
K(t, s)= & \eta_{A}(t) \eta_{A}(s) K(t, s)+\left(1-\eta_{A}(s)\right) \eta_{A}(t) K(t, s) \\
& +\left(1-\eta_{A}(t)\right) \eta_{A}(s) K(t, s)+\left(1-\eta_{A}(t)\right)\left(1-\eta_{A}(s)\right) K(t, s) .
\end{aligned}
$$

Accordingly, the integral operator $K$ splits into the sum

$$
K=K_{1 A}+K_{2 A}+K_{3 A}+K_{4 A} .
$$

The operator $K_{1 A}$ is compact because any integral operator with a weakly singular kernel is compact on any $L_{p}, p>1$. In its turn, the latter fact follows, e.g., from theorems about integrals of potential type proved in [10, 11, Chapter $1, \S \S 4,6]$. The compactness of $K$ is a consequence of the fact that, as $A \rightarrow+\infty$, the operators $K_{2 A}, K_{3 A}$, and $K_{4 A}$ tend to zero in norm. We shall prove this with the help of a series of estimates. 
$2^{\circ}$. We check that $\left\|K_{4 A}\right\| \rightarrow 0$. Put $\psi_{A}^{(4)}(t):=K_{4 A} \varphi, \varphi \in L_{p}\left(\mathbb{R}^{1}\right)$. Then, for $|t|>A$ we have

$$
\begin{aligned}
& \left|\psi_{A}^{(4)}(t)\right| \leq \operatorname{const} \int_{|s|>A} \frac{\|m(t)-m(s)\|}{|t-s|}|\varphi(s)| d s \\
& |\varphi(s)|:=\sqrt{\sum_{j=1}^{n}\left|\varphi_{j}(s)\right|^{2}}, \quad\|q\|=\left\|\left(q_{i j}\right)\right\|:=\sqrt{\sum_{i, j=1}^{n}\left|q_{i j}\right|^{2}} .
\end{aligned}
$$

Since $m(t) \in \operatorname{Lip} \alpha\left(\mathbb{R}^{1}\right)$, we arrive at the estimate

$$
\left|\psi_{A}^{(4)}(t)\right| \leq \text { const } \frac{1}{|t|^{\alpha}} \int_{|s|>A} \frac{1}{|s|^{\alpha}} \frac{|\varphi(s)|}{|s-t|^{1-\alpha}} d s .
$$

Obviously,

$$
\int_{|s|>A} \frac{|\varphi(s)| d s}{|s|^{\alpha}|t-s|^{1-\alpha}}=\int_{|s|>A} \frac{|\varphi(s)|}{|t-s|^{\frac{1-\alpha}{p}}} \cdot \frac{d s}{|t-s|^{\frac{1-\alpha}{p^{\prime}}}|s|^{\alpha}}
$$

where $\frac{1}{p}+\frac{1}{p^{\prime}}=1$. The Hölder inequality yields

$$
\int_{|s|>A} \leq\left(\int_{|s|>A} \frac{|\varphi(s)|^{p} d s}{|t-s|^{1-\alpha}}\right)^{\frac{1}{p}}\left(\int_{|s|>A} \frac{d s}{|t-s|^{1-\alpha}|s|^{\alpha p^{\prime}}}\right)^{\frac{1}{p^{\prime}}} .
$$

Substituting $s=|t| s^{\prime}$, we estimate the last-written integral:

$$
\left(\int_{|s|>A} \frac{d s}{|t-s|^{1-\alpha}|s|^{\alpha p^{\prime}}}\right)^{\frac{1}{p^{\prime}}} \leq \mathrm{const} \frac{1}{A^{\frac{\alpha}{p}}} .
$$

We pass to the estimation of $\left|\psi_{A}^{(4)}(t)\right|_{L_{p}}$. We have

$$
\left|\psi_{A}^{(4)}(t)\right|_{L_{p}\left(\mathbb{R}^{1}\right)}^{p} \leq \frac{\text { const }}{A^{\alpha}} \int_{|t|>A} d t \int_{|s|>A} \frac{|\varphi(s)|^{p} d s}{|t-s|^{1-\alpha}|t|^{\alpha p}} .
$$

Interchanging the order of integration and estimating the inner integral as in $\left(\mathrm{A}_{1} .8\right)$, namely,

$$
\int_{|t|>A} \frac{d t}{|t-s|^{1-\alpha}|t|^{\alpha p}} \leq \operatorname{const} \frac{1}{A^{\alpha(p-1)}}
$$

we obtain

$\left(\mathrm{A}_{1} .11\right) \quad\left\|\psi_{A}^{(4)}(t)\right\|_{L_{p}\left(\mathbb{R}^{1}\right)} \leq \mathrm{const} \frac{1}{A^{\frac{\alpha}{p}}} \frac{1}{A^{\frac{\alpha}{p^{\prime}}}}\left(\int_{|s|>A}|\varphi(s)|^{p} d s\right)^{\frac{1}{p}} \leq \frac{\mathrm{const}}{A^{\alpha}}\|\varphi(s)\|_{L_{p}\left(\mathbb{R}^{1}\right)}$,

which shows that $\left\|K_{4 A}\right\|$ tends to zero as $A \rightarrow+\infty$.

$3^{\circ}$. Our next step is to show that $\left\|K_{4 A}\right\| \underset{A \rightarrow+\infty}{\longrightarrow} 0$. Put $\psi_{A}^{(2)}(t)=K_{2 A} \varphi$; then

$$
\left|\psi_{A}^{(2)}(t)\right| \leq \mathrm{const} \int_{|s|>A} \frac{\|m(t)-m(s)\|}{|t-s|}|\varphi(s)| d s \eta_{A}(t) .
$$

First, assume that $|t|>1$. Then

$$
\left|\psi_{A}^{(2)}(t)\right| \leq \frac{\text { const }}{|t|^{\alpha}} \int_{|s|>A} \frac{1}{|s|^{\alpha}} \frac{|\varphi(s)| d s}{|s-t|^{1-\alpha}} \eta_{A}(t)
$$

This integral can be estimated as before:

$$
\left|\psi_{A}^{(2)}(t)\right| \leq \frac{\text { const }}{|t|^{\alpha}} \eta_{A}(t) \frac{1}{A^{\frac{\alpha}{p}}}\left(\int_{|s|>A} \frac{|\varphi(s)|^{p} d s}{|s-t|^{1-\alpha}}\right)^{\frac{1}{p}} .
$$


We have

$$
\left(\int_{1 \leq|t| \leq A}\left|\psi_{A}(t)\right|^{p} d t\right)^{\frac{1}{p}} \leq \frac{\text { const }}{A^{\frac{\alpha}{p}}}\left(\int_{1 \leq|t| \leq A} d t \int_{|s|>A} \frac{|\varphi(s)|^{p} d s}{|t|^{\alpha p}|s-t|^{1-\alpha}}\right)^{\frac{1}{p}} .
$$

After interchanging the order of integration, it is easy to show that the inner integral

$$
\int_{1 \leq|t| \leq A} \frac{d t}{|t|^{\alpha p}|s-t|^{1-\alpha}}
$$

is bounded for $|s|>A$. Now, $\left(\mathrm{A}_{1} .15\right)$ implies that

$$
\left(\int_{1 \leq|t| \leq A}\left|\psi_{A}(t)\right|^{p} d t\right)^{\frac{1}{p}} \leq \frac{\text { const }}{A^{\frac{\alpha}{p}}}\|\varphi\|_{L_{p}\left(\mathbb{R}^{1}\right)} .
$$

A similar (and stronger) estimate can be proved easily for the integral

$$
\left(\int_{|t| \leq 1}\left|\psi_{A}(t)\right|^{p} d t\right)^{\frac{1}{p}}
$$

Thus, $\left|\psi_{A}^{(2)}\right|_{L_{p}} \leq \frac{\text { const }}{A^{\frac{\rho}{p}}}\|\varphi\|_{L_{p}}$, and $\left\|K_{2 A}\right\| \leq \frac{\text { const }}{A^{\frac{\rho}{p}}} \underset{A \rightarrow+\infty}{\longrightarrow} 0$.

$4^{\circ}$. We turn to the estimation of $\left\|K_{3 A}\right\|$. As before, we put $\left|\psi_{A}^{(3)}\right|:=\left|K_{3 A} \varphi\right|$; then

$$
\left|\psi_{A}^{(3)}(t)\right| \leq \mathrm{const} \int_{|t| \leq A} \frac{\|m(t)-m(s)\|}{|t-s|}|\varphi(s)| d s .
$$

We estimate

$$
\hat{\psi}_{A}^{(3)}(t):=\int_{1 \leq|s| \leq A} \frac{\|m(t)-m(s)\|}{|t-s|}|\varphi(s)| d s .
$$

(It is easy to control the integral over the segment $|s| \leq 1$.) As above, we obtain the inequality

$$
\hat{\psi}_{A}^{(3)}(t) \leq \frac{\text { const }}{|t|^{\alpha}} \int_{|s|>A} \frac{1}{|s|^{\alpha}} \frac{|\varphi(s)| d s}{|s-t|^{1-\alpha}} \eta_{A}(t) .
$$

Further arguments, similar to those at step $3^{\circ}$, lead to the estimate

$$
\left(\int_{|s|>A}\left(\hat{\psi}_{A}^{(3)}(t)\right)^{p}\right)^{\frac{1}{p}} \leq \frac{\text { const }}{A^{\frac{1}{p^{\prime}}}}\|\varphi\|_{L_{p}},
$$

completing the proof of the fact that the integral operator $K$ is compact.

\section{Appendix 2. Proofs of Theorems 2.3 And 2.4}

$1^{\circ}$. We start with Theorem 2.3. Recall that $\Psi=K \Psi+\lambda$. We estimate $|K \Psi|$, assuming that $|t| \leq B$ ( $B$ is an arbitrary fixed positive number):

$$
\left(\mathrm{A}_{2} .1\right) \quad\left|\int_{\mathbb{R}^{1}} K(t, s) \Psi(s) d s\right| \leq\left(\int_{\mathbb{R}^{1}}|\Psi|^{p} d s\right)^{\frac{1}{p}}\left(\int_{\mathbb{R}^{1}}\|K(t, s)\|^{p^{\prime}} d s\right)^{\frac{1}{p^{\prime}}} ; \quad \frac{1}{p}+\frac{1}{p^{\prime}}=1 .
$$

For $|t| \leq B$ we have

$$
\|K(t, s)\| \leq \begin{cases}\frac{\text { const }}{|t-s|^{1-\alpha}}, & |s| \leq 2 B, \\ \frac{\text { const }}{|s|}, & |s| \geq 2 B .\end{cases}
$$

Since $(1-\alpha) p^{\prime}<1$ whenever $p \alpha>1$, the integral $\int_{|s| \leq 2 B}\|K(t, s)\|^{p^{\prime}} d s$ is bounded uniformly for $|t| \leq B$, whence so is the integral $\left.\int_{|s|>2 B}\|K(t, s)\|\right|^{p^{\prime}} d s$. Thus, the function $\Psi(t)$ is bounded on any finite interval. 
$2^{\circ}$. Now, let $|t|>B$; then

$$
\|K(t, s)\| \leq \begin{cases}\frac{\text { const }}{|t|}, & |s| \leq \frac{B}{2}, \\ |s|^{\alpha}|t-s|^{1-\alpha}|t|^{\alpha} & |s|>\frac{B}{2}\end{cases}
$$

We shall need the estimate

$$
\left(\int_{|s|>\frac{B}{2}}\|K(t, s)\|^{p^{\prime}} d s\right)^{\frac{1}{p^{\prime}}} \leq \mathrm{const}\left(\int_{|s|>\frac{B}{2}} \frac{d s}{|s|^{\alpha p^{\prime}}|t-s|^{p^{\prime}(1-\alpha)}}\right)^{\frac{1}{p^{\prime}}} \frac{1}{|t|^{\alpha}} .
$$

Putting

$$
J:=\int_{\frac{B}{2} \leq|s|} \frac{d s}{|s|^{p^{\prime} \alpha}|t-s|^{(1-\alpha) p^{\prime}}},
$$

in this integral we change the variables $s=|t| s^{\prime}$ :

$$
J:=\frac{1}{|t|^{p^{\prime}-1}} \int_{\frac{B}{2|t|} \leq|s|} \frac{d s}{|s|^{p^{\prime} \alpha}|s-\operatorname{sgn}|^{(1-\alpha) p^{\prime}}} .
$$

If $p^{\prime} \alpha<1$, then the integral in $\left(\mathrm{A}_{2} .6\right)$ is uniformly bounded, and if $p^{\prime} \alpha=1$, then it admits an estimate of order $O\left(|t|^{\varepsilon}\right)$ (with $\varepsilon$ arbitrarily small) and also an estimate of order $O\left(|t|^{\alpha p^{\prime}-1}\right)$. So, for $p^{\prime} \alpha>1$, we obtain

$$
J= \begin{cases}O\left(\frac{1}{\left.|t|\right|^{p^{\prime}-1}}\right), & p^{\prime} \alpha<1 \\ O\left(\frac{1}{|t|^{p^{\prime}-1-\varepsilon}}\right), & p^{\prime} \alpha=1 \\ O\left(\frac{1}{|t|^{p^{\prime}(1-\alpha)}}\right), & p^{\prime} \alpha>1\end{cases}
$$

We substitute $\left(\mathrm{A}_{2} .7\right)$ in $\left(\mathrm{A}_{2} .1\right)$. Since $|\Psi|$ is bounded if $|t| \leq$ const, we arrive at an inequality valid for $|t|>\frac{B}{2}$ :

$$
\left|\int_{\mathbb{R}^{1}} K(t, s) \Psi(s) d s\right| \leq \mathrm{const} \begin{cases}\frac{1}{|t|^{\alpha+1-\frac{1}{p^{\prime}}}}=\frac{1}{|t|^{\alpha+\frac{1}{p}}}, & p^{\prime} \alpha<1 \\ \frac{1}{|t|^{\alpha+\frac{1}{p}-\varepsilon}}, & p^{\prime} \alpha=1 \\ \frac{1}{|t|}, & p^{\prime} \alpha>1 .\end{cases}
$$

$3^{\circ}$. We have obtained the estimate

$$
\Psi(t)=O\left(\frac{1}{1+|t|^{\beta}}\right)+\lambda(t), \quad t \in \mathbb{R}^{1},
$$

where $\beta$ is equal to 1 , or $\alpha+\frac{1}{p}$, or $\alpha+\frac{1}{p}-\varepsilon$. Observe that for $p^{\prime} \alpha<1$ we have $\alpha+\frac{1}{p}<1$.

If $\beta<1$, then, after one or several iterations, we can replace $\left(\mathrm{A}_{2} .9\right)$ with a relation of the form

$$
\Psi(t)=O\left(\frac{1}{1+|t|}\right)+\tilde{\lambda}(t), \quad t \in \mathbb{R}^{1}, \quad \tilde{\lambda} \in \operatorname{Lip} \alpha\left(\mathbb{R}^{1}\right), \quad \tilde{\lambda}(\infty)=0
$$

Actually, the substitution of $\lambda(t)$, or of another function belonging to $\operatorname{Lip} \alpha\left(\mathbb{R}^{1}\right)$ and vanishing at $t=\infty$, into the integral operator $\int_{\mathbb{R}^{1}} K(t, s)(\cdots) d s$, results in a function again belonging to $\in \operatorname{Lip} \alpha\left(\mathbb{R}^{1}\right)$ and vanishing at $t=\infty$, and the quantity $\frac{1}{p}-\varepsilon$ is added to the exponent $\beta$ in the estimate $O\left(\frac{1}{1+|t|^{\beta}}\right)$ for the function obtained. This leads to a term that decays as $O\left(\frac{1}{|t|^{\beta+\frac{1}{p}-\varepsilon}}\right)$. Repeating (if needed) this process, we can obtain a 
function that decays as $O\left(\frac{1}{1+|t| \gamma}\right)$, where $\gamma p^{\prime}>1$. Then our integral operator applied to such a function admits an estimate of order $O\left(\frac{1}{1+|t|}\right)$. Thus, we have proved that

$$
\Psi(t)=O\left(\frac{1}{1+|t|}\right)+\tilde{\lambda}(t), \quad \tilde{\lambda}(t) \in \operatorname{Lip} \alpha\left(\mathbb{R}^{1}\right), \quad \tilde{\lambda}(\infty)=0 .
$$

$4^{\circ}$. Now we show that $\Psi(t) \in \operatorname{Lip} \alpha^{\prime}(|t| \leq B)$, where $0<\alpha^{\prime}<\alpha$, i.e., $\Psi(t)$ belongs to Lip $\alpha^{\prime}$ on any finite interval.

Let $|t| \leq B$. In the integral $\int_{\mathbb{R}^{1}} K(t, s) \Psi(s) d s$, we split the domain of integration into two sets: $|s| \leq 4 B$ and $|s|>4 B$. Then

$$
\Psi(t)=\int_{|s| \leq 4 B} K(t, s) \Psi(s) d s+\int_{|s|>4 B} K(t, s) \Psi(s) d s+\lambda(t) .
$$

The second and third terms belong to $\operatorname{Lip} \alpha(|t| \leq B)$. It remains to analyze the Lipschitz property for the first term.

For $|t| \leq B$ and $|\Delta t| \leq \frac{B}{2}$, we estimate

$$
\begin{array}{r}
\left|\int_{|s| \leq 4 B} K(t+\Delta t, s) \Psi(s) d s-\int_{|s| \leq 4 B} K(t, s) \Psi(s) d s\right| \\
=\mid \int_{|s| \leq 4 B} m^{-1}(t+\Delta t) \frac{(m(t+\Delta t)-m(s))}{t+\Delta t-s} \Psi(s) d s \\
-\int_{|s| \leq 4 B} m^{-1}(t) \frac{m(t)-m(s)}{t-s} \Psi(s) d s \mid .
\end{array}
$$

Since the function $m^{-1}(t)$ is Lipschitz, it suffices to estimate the quantity

$$
J:=\left|\int_{|s| \leq 4 B} \frac{(m(t+\Delta t)-m(s))}{t+\Delta t-s} \Psi(s) d s-\int_{|s| \leq 4 B} \frac{m(t)-m(s)}{t-s} \Psi(s) d s\right| .
$$

Obviously,

$$
J \leq\left|\int_{|t-s| \leq 3|\Delta t|}\right|+\left|\int_{3|\Delta t| \leq|t-s| \leq 2 B}\right|+\left|\int_{\{|s| \leq 4 B\} \backslash\{s:|t-s| \leq 2 B\}}\right| .
$$

Since $|t+\Delta t-s|$ and $|t-s|$ in the last integral have a positive lower bound, a Lipschitz estimate of this integral can be obtained easily. Since $\Psi(s)$ is bounded, we have

$$
\begin{aligned}
& \left|\int_{|t-s| \leq 3|\Delta t|} \frac{\|m(t+\Delta t)-m(s)\||\Psi(s)| d s}{|t+\Delta t-s|}\right| \\
& \leq \text { const } \max |\Psi(s)| \int_{|t-s| \leq 3|\Delta t|}|t+\Delta t-s|^{\alpha-1} d s .
\end{aligned}
$$

Substituting $s-t=\frac{x}{|\Delta t|}$, for this integral we obtain an estimate of the form $O\left(|\Delta t|^{\alpha}\right)$.

The integral

$$
\left|\int_{|t-s| \leq 3|\Delta t|} \frac{m(t)-m(s)}{t-s} \Psi(s) d s\right|
$$

is estimated similarly. The estimate is also of the form $O\left(|\Delta t|^{\alpha}\right)$.

We turn to the integral

We have

$$
J_{1}:=\int_{3|\Delta t| \leq|t-s| \leq 2 B} .
$$

$\left(\mathrm{A}_{2} .17\right)$

$$
J_{1}=\left|\int_{3|\Delta t| \leq|t-s| \leq 2 B}\left(\frac{(m(t+\Delta t)-m(t))}{t+\Delta t-s} \Psi(s)+\Delta t \frac{m(t)-m(s)}{(t+\Delta t-s)(t-s)} \Psi(s)\right) d s\right| .
$$


Since $\|(m(t)-m(s))\| \leq$ const $|t-s|^{\alpha}$, the function $\Psi(s)$ is bounded, and $|t-s| \geq 3|\Delta t|$, it follows that the integral of the second term admits an estimate of order $O\left(|\Delta t|^{\alpha}\right)$. The integral of the first term is estimated as follows:

$$
\begin{aligned}
& O\left(|\Delta t|^{\alpha}\right)\left|\int_{3|\Delta t| \leq|t-s| \leq 2 B} \frac{\Psi(s) d s}{t+\Delta t-s}\right| \\
& \quad \leq O\left(|\Delta t|^{\alpha}\right) \operatorname{const}\left(1+\left|\log \frac{1}{|\Delta t|}\right|\right) \leq O\left(|\Delta t|^{\alpha}\right) \operatorname{const}_{\varepsilon}|\Delta t|^{-\varepsilon},
\end{aligned}
$$

where $\varepsilon>0$ is arbitrarily small.

Thus, we have proved that $\Psi(t)$ is Lipschitz with exponent $0<\alpha^{\prime}<\alpha$ on any finite segment.

$5^{\circ}$. We show that $\Psi(t) \in \operatorname{Lip} \alpha(|t| \leq B)$. It suffices to prove that the integral in $\left(\mathrm{A}_{2} .18\right)$ is bounded. We have shown that $\Psi(t)$ is Lipschitz on any finite interval, but with exponent $\alpha^{\prime}<\alpha$. This allows us to estimate the integral mentioned above, because

$$
\begin{aligned}
\int_{3|\Delta t| \leq|t-s| \leq 2 B} \frac{\Psi(s)}{t+\Delta t-s} d s= & \int_{3|\Delta t| \leq|t-s| \leq 2 B} \frac{(\Psi(s)-\Psi(t+\Delta t))}{t+\Delta t-s} d s \\
& +\Psi(t+\Delta t) \int_{3|\Delta t| \leq|t-s| \leq 2 B} \frac{d s}{t+\Delta t-s} .
\end{aligned}
$$

Here the first integral on the right is bounded because $\Psi(t)$ is Lipschitz, while the second can be calculated explicitly, and its boundedness is established easily.

$6^{\circ}$. For the proof of Theorem 2.4 it remains to check that $\Psi(t)$ is Lipschitz for $|t|>B$ ( $B$ is an arbitrary positive number). For this, we introduce two real cut-off functions $\eta$ and $\eta_{1}=1-\eta$ continuously differentiable on $\mathbb{R}$ and such that

$$
\eta(s)= \begin{cases}1, & |s| \leq \frac{B}{4}, \\ 0, & |s|>\frac{B}{2} .\end{cases}
$$

Then the integral $\int_{\mathbb{R}^{1}} K(t, s) \Psi(s) d s$ can be written as a sum:

$$
\begin{aligned}
\int_{\mathbb{R}^{1}} K(t, s) \Psi(s) d s & =\int_{\mathbb{R}^{1}} K(t, s)\left(\eta+\eta_{1}\right) \Psi(s) d s \\
& =\int_{|s| \leq \frac{B}{2}} K(t, s) \eta(s) \Psi(s) d s+\int_{|s| \geq \frac{B}{4}} K(t, s) \eta_{1}(s) \Psi(s) d s .
\end{aligned}
$$

Obviously, the integral $\int_{|s| \leq \frac{B}{2}}$ is Lipschitz. Moreover,

$$
\int_{|s| \leq \frac{B}{4}}=\frac{1}{t} \tilde{\Psi}(t)
$$

where $\tilde{\Psi}(t)$ is Lipschitz for $|t|>B$, which follows immediately from the form of $K(t, s)$. The integral equation takes the form

$$
\left(\mathrm{A}_{2} .21\right) \Psi(t)=\int_{|s|>\frac{B}{4}} K(t, s) \eta_{1}(s) \Psi(s) d s+\lambda_{1}(t), \quad \lambda_{1} \in \operatorname{Lip} \alpha\left(|t| \geq \frac{B}{4}\right), \quad \lambda_{1}(\infty)=0 .
$$

Lemma 1. If $m$ is sufficiently large and $|t| \geq$ const $>0$, then $K_{1}^{m} \lambda_{1}=\frac{\Lambda(t)}{t}$, where $K_{1}(\cdots)=\int_{|s|>\frac{B}{4}} K(t, s) \eta_{1}(s) \Psi(s) d s$ and $\Lambda(t) \in \operatorname{Lip} \alpha(|t| \geq$ const $>0)$.

The proof reduces, first, to the estimate $K^{m^{\prime}} \lambda=O\left(\frac{1}{t}\right)$, valid for large $m^{\prime}$ and obtained by consecutive estimates in the spirit of those at the beginning of this Appendix. Next, in the integral

$$
K_{1}^{m^{\prime}+1} \lambda_{1}=\int_{\frac{B}{4}}^{\infty} K(t, s) \eta_{1}(s) K_{1}^{m^{\prime}} \lambda_{1} d s
$$


we put $t^{\prime}=\frac{1}{t}$ and $s^{\prime}=\frac{1}{s}$, proving that $K_{1}^{m^{\prime}+1} \lambda_{1}=t^{\prime} \Lambda_{1}\left(t^{\prime}\right)$ with $\Lambda_{1}\left(t^{\prime}\right) \in \operatorname{Lip} \alpha^{\prime}\left(\left|t^{\prime}\right| \leq \frac{4}{B}\right)$, $\alpha^{\prime}<\alpha$. After that, estimates similar to those in Subsection $5^{\circ}$ show that the function $K^{m+2} \lambda_{1}$ possesses the required properties.

$7^{\circ}$. Now we put

$$
\Psi=\Psi_{m}+\sum_{j=0}^{m-1} K_{1}^{j} \lambda_{1} .
$$

It is easily seen that $\Psi_{m}=K^{m} \Psi$. For $m$ large, we have $K^{m} \Psi=O\left(\frac{1}{1+|t|}\right)$, and

$$
\Psi_{m}=K_{1} \Psi_{m}+K_{1}^{m} \lambda_{1} .
$$

Passing in this equation to the variables $s^{\prime}=\frac{1}{s}$ and $t^{\prime}=\frac{1}{t}$, and putting

$$
\psi_{m}\left(t^{\prime}\right)=\frac{\Psi_{m}\left(t^{\prime}\right)}{t^{\prime}}, \quad K_{1}^{m} \lambda_{1}=\frac{\Lambda_{1}\left(t^{\prime}\right)}{t^{\prime}},
$$

we arrive at an equation on the finite segment $\left|s^{\prime}\right| \leq \frac{4}{B}$ with a Lipschitz right-hand side and with a kernel of the form

$$
\pm \frac{1}{2 \pi i} m_{1}^{-1}\left(t^{\prime}\right) \frac{m_{1}\left(t^{\prime}\right)-m_{1}\left(s^{\prime}\right)}{t^{\prime}-s^{\prime}} \eta_{2}\left(s^{\prime}\right), \quad m_{1}\left(t^{\prime}\right)=m\left(\frac{1}{t^{\prime}}\right), \quad \eta_{2}\left(s^{\prime}\right)=\eta_{1}\left(\frac{1}{s^{\prime}}\right),
$$

in the class of functions bounded on the segment $\left|t^{\prime}\right| \leq \frac{4}{B}$. For such equations, the Lipschitz property of solutions was proved at the beginning of this Appendix. Returning to the variable $t$, for $|t|>\frac{B}{4}$ we obtain

$$
\Psi=\Psi_{m}+\sum_{j=1}^{m-1} K_{1}^{j} \lambda_{1}=\frac{1}{t} \Psi_{m}\left(t^{\prime}\right)+\sum_{j=1}^{m-1} K_{1}^{j} \lambda_{1} .
$$

We recall that the functions $\Psi_{m}\left(t^{\prime}\right)$ and $K_{1}^{j} \lambda_{1}$ are Lipschitz, $t^{\prime}=\frac{1}{t}$, and $K_{1}^{j} \lambda_{1}=O\left(\frac{1}{|t| \alpha}\right)$ for $j=1, \ldots, m-1$ (see Subsection $3^{\circ}$ ). Therefore,

$$
\Psi \in \operatorname{Lip} \alpha\left(|t| \geq \frac{B}{4}\right), \quad \Psi(t)=O\left(|t|^{-\alpha}\right) .
$$

$8^{\circ}$. We pass to the proof of Theorem 2.4. First, assume that $\alpha p>1$. Then the proof of Theorem 2.3 implies that $\Psi=O\left(\frac{1}{1+|t|^{\beta}}\right), \beta>0$. Performing several iterations, as before, we obtain $\Psi=O\left(\frac{1}{1+|t|}\right)$. Returning to the equation

$$
\Psi=\int_{\mathbb{R}^{1}} K(t, s) \Psi(s) d s,
$$

we let $\eta$ and $\eta_{1}$ be the cut-off functions introduced in Subsection $6^{\circ}$. Again, we split $\int_{\mathbb{R}^{1}}$ into a sum:

$$
\int_{\mathbb{R}^{1}} K(t, s) \Psi(s) d s=\int_{\mathbb{R}^{1}} K(t, s) \eta(s) \Psi(s) d s+\int_{\mathbb{R}^{1}} K(t, s) \eta_{1}(s) \Psi(s) d s .
$$

This leads to the identity

$$
\Psi(t)=\int_{|s| \geq \frac{B}{4}} K(t, s) \eta_{2}(s) \Psi(s) d s+\frac{\mu(t)}{t},
$$

where $\mu(t) \in \operatorname{Lip} \alpha\left(|t| \geq \frac{B}{4}\right)$. 
Introducing again the variables $t^{\prime}=\frac{1}{t}, s^{\prime}=\frac{1}{s}$, we reduce the problem to the case of an equation in the class of bounded functions and with right-hand side belonging to $\operatorname{Lip} \alpha\left(|t| \leq \frac{4}{B}\right)$. Indeed, if we take $\psi\left(t^{\prime}\right)=\frac{\Psi_{1}\left(t^{\prime}\right)}{t^{\prime}}$ with $\Psi_{1}\left(t^{\prime}\right)=\Psi\left(\frac{1}{t^{\prime}}\right)=\Psi(t)$ for the role of the new unknown function, we arrive at the equation

$$
\begin{gathered}
\psi\left(t^{\prime}\right)=\varepsilon \frac{1}{2 \pi i} \int_{\left|s^{\prime}\right| \leq \frac{4}{B}} m_{1}^{-1}\left(t^{\prime}\right) \frac{m_{1}\left(t^{\prime}\right)-m_{1}\left(s^{\prime}\right)}{t^{\prime}-s^{\prime}} \eta_{2}\left(s^{\prime}\right) \psi\left(s^{\prime}\right) d s^{\prime}+\mu_{1}\left(t^{\prime}\right), \\
\mu_{1}\left(t^{\prime}\right)=\mu\left(\frac{1}{t^{\prime}}\right) .
\end{gathered}
$$

(The notation is as in Subsection $7^{\circ}$, and $\varepsilon$ is equal to +1 or -1 .) Since equation $\left(A_{2} .22\right)$ may only have Lipschitz solutions, we have

$$
\Psi(t)=t^{\prime} \psi\left(t^{\prime}\right)=\frac{1}{t} \psi\left(t^{\prime}\right), \quad \psi\left(t^{\prime}\right) \in \operatorname{Lip} \alpha\left(\left|t^{\prime}\right| \leq \frac{4}{B}\right) .
$$

Thus, the desired expression for $\Psi(t)$ is proved (see the statement of Theorem 2.4).

$9^{\circ}$. It remains to show that $\Psi \in L_{p}\left(\mathbb{R}^{1}\right)$ with $\alpha p>1$. This follows from the fact that, for $\alpha p<1$, the equation implies that $\Psi$ is integrable with a larger exponent $q^{*}>p$. If $\alpha q^{*}>1$, we arrive at the desired statement. If $\alpha q^{*} \leq 1$, then this process should be repeated; as a result, $\Psi$ will be integrable with an exponent $Q$ such that $\alpha Q>1$. We pass to the corresponding arguments.

Let $B$ be an arbitrary positive constant, and let $|t| \leq B$. Estimates $\left(A_{2} .2\right)$ imply that

$$
|\Psi(t)| \leq \mathrm{const}\left(\int_{|s| \leq 2 B} \frac{|\Psi(s)| d s}{|t-s|^{1-\alpha}}+\int_{|s|>2 B} \frac{|\Psi(s)| d s}{|s|}\right) .
$$

The second integral is bounded, and the first is a "potential type integral", viewed as a function of $t$ for $|t| \leq 2 B$, and as such belongs to $L_{q^{*}}$, where $q^{*}$ is any positive number exceeding 1 and satisfying

$$
\frac{1}{q^{*}}>\frac{1}{p}-\alpha
$$

(for the proof, see [11, Chapter $1, \S 6]$ ).

$10^{\circ}$. Now, let $|t|>B$. We want to prove that

$$
\Psi(t) \in L_{q^{*}}(|t|>B) .
$$

We start with estimates $\left(A_{2} .3\right)$, which imply that, for $|t|>B$,

$$
|\Psi(t)| \leq \operatorname{const}\left(\int_{|s| \leq \frac{B}{2}} \frac{|\Psi(s)| d s}{|t|}+\int_{|s|>\frac{B}{2}} \frac{|\Psi(s)| d s}{|t|^{\alpha}|t-s|^{1-\alpha}|s|^{\alpha}}\right) .
$$

The first term is integrable with any exponent exceeding 1 . In order to estimate the second integral 6 we note that inequality $\left(\mathrm{A}_{2} .24\right)$ implies the existence of a positive number $\varepsilon$, which may be assumed as small as we wish, such that

$$
\frac{1}{q^{*}}=\frac{1}{p}-\alpha+2 \varepsilon \Leftrightarrow 1-\alpha=\left(\frac{1}{q^{*}}-\varepsilon\right)+\left(\frac{1}{p^{\prime}}-\varepsilon\right) ; \quad \frac{1}{p}+\frac{1}{p^{\prime}}=1 .
$$

${ }^{6}$ Largely, these estimates are similar to those in Chapter 1 of the book [1]. 
Therefore,

$$
\begin{aligned}
J & :=\int_{|s|>\frac{B}{2}} \frac{|\Psi| d s}{|t|^{\alpha}|t-s|^{1-\alpha}|s|^{\alpha}} \\
= & \frac{1}{|t|^{\alpha}} \int_{|s|>\frac{B}{2}} \frac{|\Psi|^{\frac{p}{q^{*}}}}{|t-s|^{\frac{1}{q^{*}}-\varepsilon}}|\Psi|^{p\left(\frac{1}{p}-\frac{1}{q^{*}}\right)} \frac{1}{|t-s|^{\frac{1}{p^{*}}-\varepsilon}|s|^{\alpha}} d s \\
\leq & \frac{1}{|t|^{\alpha}}\left(\int\left(\frac{|\Psi|^{\frac{p}{q^{*}}}}{|t-s|^{\frac{1}{q^{*}}-\varepsilon}}\right)^{\frac{1}{\mu_{1}}} d s\right)^{\mu_{1}}\left(\int\left(|\Psi|^{p\left(\frac{1}{p}-\frac{1}{q^{*}}\right)}\right)^{\frac{1}{\mu_{2}}} d s\right)^{\mu_{2}} \\
& \times\left(\int\left(\frac{1}{|t-s|^{\frac{1}{p^{\prime}}-\varepsilon}|s|^{\alpha}}\right)^{\frac{1}{\mu_{3}}} d s\right)^{\mu_{3}}, \\
\mu_{1} & =\frac{1}{q^{*}}, \quad \mu_{2}=\frac{1}{p}-\frac{1}{q^{*}}, \quad \mu_{3}=\frac{1}{p^{\prime}} .
\end{aligned}
$$

(We have applied the generalized Hölder inequality.) Thus,

$$
J \leq \frac{\text { const }}{|t|^{\alpha}}\left(\int_{|s|>\frac{B}{2}} \frac{|\Psi|^{p} d s}{|t-s|^{1-q^{*} \varepsilon}}\right)^{\frac{1}{q^{*}}}\|\Psi\|_{L_{p}}^{\frac{1}{p}-\frac{1}{q^{*}}}\left(\int_{|s|>\frac{B}{2}} \frac{d s}{|t-s|^{1-p^{\prime} \varepsilon}|s|^{p^{\prime} \alpha}}\right)^{\frac{1}{p^{\prime}}}
$$

The last factor can be estimated easily:

$$
\left(\int_{|s|>\frac{B}{2}}\right)^{\frac{1}{p^{\prime}}}= \begin{cases}O\left(\frac{1}{|t|^{\alpha-\varepsilon}}\right), & \alpha p^{\prime}<1, \\ O\left(\frac{1}{|t|^{\alpha-\varepsilon^{\prime}}}\right), & \alpha p^{\prime}=1, \quad 0<\varepsilon^{\prime}<\epsilon, \\ O\left(\frac{1}{|t|^{\frac{1}{p^{\prime}}-\varepsilon}}\right), & \alpha p^{\prime}>1 .\end{cases}
$$

Now we need to estimate $\|J\|_{L_{q^{*}}(|t|>B)}$. The integral $\int_{|t|>B} J^{q^{*}} d s$ is estimated easily: using $\left(A_{2} .29\right)$ and interchanging the integrals, after simple transformations we obtain

$$
\left(\int_{|t|>B} J^{q^{*}}\right)^{\frac{1}{q^{*}}}=\|J\|_{L_{q^{*}}(|t|>B)} \leq \mathrm{const}\|\Psi\|_{L_{p}(|t|>B)} .
$$

Collecting the estimates of $\Psi(t)$ obtained in Subsections $9^{\circ}$ and $10^{\circ}$, we arrive at the desired result:

$$
\Psi(t) \in L_{q^{*}}\left(\mathbb{R}^{1}\right)
$$

where $q^{*}$ is any number exceeding 1 and such that $\frac{1}{q^{*}}>\frac{1}{p}-\alpha$.

If among the numbers $q^{*}$ satisfying $\frac{1}{q^{*}}>\frac{1}{p}-\alpha$ there exists $q^{*}\left(q^{*}>p\right)$ such that $\frac{1}{q^{*}}-\alpha<0$, then the proof of Theorem 2.4 is complete. If there is no such $q^{*}$, then we can repeat similar arguments to obtain $q_{1}^{*}$ such that

$$
\Psi(t) \in L_{q_{1}^{*}}\left(\mathbb{R}^{1}\right)
$$

and $\frac{1}{q_{1}^{*}}>\frac{1}{q^{*}}-\alpha$. After several steps, we shall arrive at an exponent $Q$ satisfying $\frac{1}{Q}-\alpha<0$. This finishes the proof of Theorem 2.4. 


\section{Appendix 3. Proof of Theorem 2.5}

We aim at proving the following theorem 7

Theorem. Let $\omega(t) \in \operatorname{Lip} \alpha\left(\mathbb{R}^{1}\right)$, let $\omega(\infty)=0$, and let $f(z)$ be the Cauchy integral

$$
f(z)=\frac{1}{2 \pi i} \int_{\mathbb{R}^{1}} \frac{\omega(s) d s}{s-z} .
$$

Then $f(z) \in \operatorname{Lip} \alpha\left(\Pi_{ \pm}\right)$. If, moreover, for $|t| \geq B=$ const $>0$ we have $\omega(t)=\frac{\omega_{1}(t)}{t}$ with $\omega_{1}(t) \in \operatorname{Lip} \alpha(|t| \geq B)$, then for $|z| \geq B$ we have $f(z)=\frac{1}{z} f_{1}(z)$ with $f_{1}(z) \in$ $\operatorname{Lip} \alpha\left(\Pi_{ \pm} \cap|z| \geq B\right)$.

Proof. $1^{\circ}$. Consider the case of $z \in \Pi_{+}$( $\Pi_{-}$is treated similarly). Suppose $z^{\prime}, z^{\prime \prime} \in \Pi_{+}$, $\operatorname{Im} z^{\prime} \geq \beta=$ const, $\operatorname{Im} z^{\prime \prime} \geq \beta=$ const, $\left|\operatorname{Re} z^{\prime}\right| \leq B$, and $\left|\operatorname{Re} z^{\prime \prime}\right| \leq B$, where $B$ and $\beta$ are arbitrary positive constants. Then

$$
\left|f\left(z^{\prime}\right)-f\left(z^{\prime \prime}\right)\right| \leq\left|z^{\prime}-z^{\prime \prime}\right| \max \left|f^{\prime}(z)\right|, \quad z \in \Pi_{+} .
$$

The modulus of the derivative is estimated easily:

$$
\left|f^{\prime}(z)\right|=\left|\frac{1}{2 \pi i} \int_{\mathbb{R}^{1}} \frac{\omega(t) d t}{(t-z)^{2}}\right| \leq \frac{\max |\omega(t)|}{2 \pi} \max \int_{\mathbb{R}^{1}} \frac{d t}{|t-z|^{2}} \leq \frac{\max |\omega(t)|}{2 \pi} \frac{\pi}{\beta} .
$$

Inequalities $\left(A_{3} .2\right)$ and $\left(A_{3} .3\right)$ imply the inequality

$$
\left|f\left(z^{\prime}\right)-f\left(z^{\prime \prime}\right)\right| \leq \text { const }\left|z^{\prime}-z^{\prime \prime}\right| .
$$

To prove that $f(z)$ is Lipschitz in the rectangle $|\operatorname{Re} z| \leq B, 0<\operatorname{Im} z \leq$ const, it suffices to check the required estimates only in a boundary strip adjoining the real axis.

For definiteness, let $\operatorname{Im} z^{\prime}>\operatorname{Im} z^{\prime \prime}>0$ and $\operatorname{Re} z^{\prime \prime}<\operatorname{Re} z^{\prime}$. Then, obviously,

$$
\left|f\left(z^{\prime}\right)-f\left(z^{\prime \prime}\right)\right| \leq\left|f\left(z^{\prime}\right)-f\left(z_{0}^{\prime \prime}\right)\right|+\left|f\left(z_{0}^{\prime \prime}\right)-f\left(z^{\prime \prime}\right)\right|, \quad z_{0}^{\prime \prime}=\operatorname{Re} z^{\prime}+i \operatorname{Im} z^{\prime \prime} .
$$

Thus, it suffices to prove the Lipschitz property in the case where the points $z^{\prime}$ and $z^{\prime \prime}$ lie on a line perpendicular to $\mathbb{R}^{1}$ or on a line parallel to $\mathbb{R}^{1}$.

$2^{\circ}$. Suppose that $z^{\prime}$ and $z^{\prime \prime}$ lie on a line perpendicular to $\mathbb{R}^{1}$; then

$$
\begin{gathered}
f\left(z^{\prime}\right)-f\left(z^{\prime \prime}\right)=\frac{1}{2 \pi i}\left(\int_{\mathbb{R}^{1}} \frac{\omega(t) d t}{t-t_{0}-s^{\prime} i}-\int_{\mathbb{R}^{1}} \frac{\omega(t) d t}{t-t_{0}-s^{\prime \prime} i}\right), \\
s^{\prime}=\operatorname{Im} z^{\prime}, \quad s^{\prime \prime}=\operatorname{Im} z^{\prime \prime}, \quad t_{0}=\operatorname{Re} z^{\prime}=\operatorname{Re} z^{\prime \prime} .
\end{gathered}
$$

This identity implies

$$
\begin{aligned}
\left|f\left(z^{\prime}\right)-f\left(z^{\prime \prime}\right)\right| \leq \frac{1}{2 \pi} & \left(\left|\int_{\left|t-t_{0}\right| \geq 3 B} \frac{\omega(t) d t}{t-t_{0}-s^{\prime} i}-\int_{\left|t-t_{0}\right| \geq 3 B} \frac{\omega(t) d t}{t-t_{0}-s^{\prime \prime} i}\right|\right. \\
& \left.+\left|\int_{\left|t-t_{0}\right|<3 B} \frac{\omega(t) d t}{t-t_{0}-s^{\prime} i}-\int_{\left|t-t_{0}\right|<3 B} \frac{\omega(t) d t}{t-t_{0}-s^{\prime \prime} i}\right|\right) .
\end{aligned}
$$

Here, only the integrals over the segment $\left|t-t_{0}\right|<3 B$ present some difficulty. It suffices to estimate

$$
\frac{1}{2 \pi}\left|\int_{\left|t-t_{0}\right|<3 B} \frac{\left(\omega(t)-\omega\left(t_{0}\right)\right) d t}{t-t_{0}-s^{\prime} i}-\int_{\left|t-t_{0}\right|<3 B} \frac{\left(\omega(t)-\omega\left(t_{0}\right)\right) d t}{t-t_{0}-s^{\prime \prime} i}\right| .
$$

\footnotetext{
${ }^{7}$ A similar theorem was proved by N. I. Muskhelishvili for the case of a finite contour; see [6, Chapter 1]. His proof, based on the maximum principle, cannot be directly carried over to the case of an infinite contour.
} 
Let $s^{\prime}-s^{\prime \prime}=\left|z^{\prime}-z^{\prime \prime}\right|:=|\Delta z|$. Obviously, we have

$$
\begin{aligned}
\frac{1}{2 \pi} \int_{\left|t-t_{0}\right|<|\Delta z|} \frac{\left|\omega(t)-\omega\left(t_{0}\right)\right| d t}{\left|t-t_{0}-s^{\prime} i\right|} \leq \mathrm{const}|\Delta z|^{\alpha}, \\
\frac{1}{2 \pi} \int_{\left|t-t_{0}\right|<|\Delta z|} \frac{\left|\omega(t)-\omega\left(t_{0}\right)\right| d t}{\left|t-t_{0}-s^{\prime \prime} i\right|} \leq \mathrm{const}|\Delta z|^{\alpha} .
\end{aligned}
$$

It remains to estimate

$$
\frac{1}{2 \pi}\left|\int_{|\Delta z| \leq\left|t-t_{0}\right|<3 B}\left(\frac{\left(\omega(t)-\omega\left(t_{0}\right)\right)}{t-t_{0}-s^{\prime} i}-\frac{\left(\omega(t)-\omega\left(t_{0}\right)\right)}{t-t_{0}-s^{\prime \prime} i}\right) d t\right| .
$$

Obviously,

$$
\begin{aligned}
& \frac{1}{2 \pi}\left|\int_{|\Delta z| \leq\left|t-t_{0}\right|<3 B} \frac{\left(\omega(t)-\omega\left(t_{0}\right)\right) i\left(s^{\prime \prime}-s^{\prime}\right) d t}{\left(t-t_{0}-s^{\prime} i\right)\left(t-t_{0}-s^{\prime \prime} i\right)}\right| \\
& \quad \leq \mathrm{const} \int_{|\Delta z| \leq\left|t-t_{0}\right|<3 B} \frac{|\Delta z|\left|t-t_{0}\right|^{\alpha} d t}{\sqrt{\left(t-t_{0}\right)^{2}+s^{\prime 2}} \sqrt{\left(t-t_{0}\right)^{2}+s^{\prime \prime 2}}} .
\end{aligned}
$$

Substituting $t-t_{0}=x$, we obtain

$$
\text { const } \int_{|x| \geq|\Delta z|} \frac{|x|^{\alpha} d x}{x^{2}}|\Delta z|=\text { const }|\Delta z|^{\alpha} .
$$

$3^{\circ}$. Suppose that $z^{\prime}$ and $z^{\prime \prime}$ lie on a line parallel to the real axis: $z^{\prime}=\beta+i \gamma$, $z^{\prime \prime}=z^{\prime}+\Delta z, \gamma>0, \Delta z$ is real. As above, it suffices to estimate

$$
\frac{1}{2 \pi}\left|\int_{|t-\beta| \leq 3 B}\left(\frac{\omega(t)-\omega(\beta+\Delta z)}{t-\beta-i \gamma-\Delta z}-\frac{\omega(t)-\omega(\beta)}{t-\beta-i \gamma}\right) d t\right| .
$$

First, we split the integral into two:

$$
\int_{|t-\beta| \leq 3|\Delta z|}+\int_{3|\Delta z| \leq|t-\beta| \leq 3 B} .
$$

The first integral is estimated easily:

$$
\begin{aligned}
\left|\int_{|t-\beta| \leq 3|\Delta z|}\right| \leq & \frac{1}{2 \pi} \int_{|t-\beta| \leq 3|\Delta z|} \frac{|\omega(t)-\omega(\beta+\Delta z)|}{|t-\beta-i \gamma-\Delta z|} d t \\
& +\frac{1}{2 \pi} \int_{|t-\beta| \leq 3|\Delta z|} \frac{|\omega(t)-\omega(\beta)|}{|t-\beta-i \gamma|} d t \\
\leq & \text { const }|\Delta z|^{\alpha}+\text { const }|\Delta z|^{\alpha} .
\end{aligned}
$$

The second integral is equal to

$$
\begin{aligned}
& \frac{1}{2 \pi} \int_{3|\Delta z| \leq|t-\beta| \leq 3 B} \frac{(\omega(t)-\omega(\beta+\Delta z))(t-\beta-i \gamma)-(\omega(t)-\omega(\beta))(t-\beta-i \gamma-\Delta z)}{(t-\beta-i \gamma-\Delta z)(t-\beta-i \gamma)} d t \\
& \quad=\frac{1}{2 \pi} \int_{3|\Delta z| \leq|t-\beta| \leq 3 B} \frac{(\omega(t)-\omega(\beta)) \Delta z+(\omega(\beta)-\omega(\beta+\Delta z))(t-\beta-i \gamma)}{(t-\beta-i \gamma-\Delta z)(t-\beta-i \gamma)} d t .
\end{aligned}
$$

In its turn, this integral splits into two:

$$
\begin{aligned}
& \frac{1}{2 \pi} \int_{3|\Delta z| \leq|t-\beta| \leq 3 B} \frac{(\omega(t)-\omega(\beta)) \Delta z d t}{(t-\beta-i \gamma-\Delta z)(t-\beta-i \gamma)} \\
& \quad+\frac{1}{2 \pi} \int_{3|\Delta z| \leq|t-\beta| \leq 3 B} \frac{(\omega(\beta)-\omega(\beta+\Delta z)) d t}{(t-\beta-i \gamma-\Delta z)} .
\end{aligned}
$$

The first term is easily estimated by $O\left(|\Delta z|^{\alpha}\right)$. The second is calculated explicitly, and the result is also of order $O\left(|\Delta z|^{\alpha}\right)$. In conclusion we note that the corresponding 
estimates for $\gamma=0$ have been performed in many publications (see, e.g., [6, Chapter 1]). The case where $\gamma \neq 0$ does not lead to complications.

$4^{\circ}$. Suppose that $|t| \geq$ const $>0,|z| \geq$ const $>0, z \in \Pi_{+}$or $z \in \Pi_{-}$. Changing the variables, we obtain the integral

$$
\frac{1}{2 \pi i} \int_{\left|t^{\prime}\right| \leq B^{\prime}} \frac{\omega\left(t^{\prime}\right)}{\frac{1}{t^{\prime}}-\frac{1}{z^{\prime}}} \frac{d t^{\prime}}{\left(-t^{\prime 2}\right)}=\frac{1}{2 \pi i} \int_{\left|t^{\prime}\right| \leq B^{\prime}} \frac{\omega\left(t^{\prime}\right) z^{\prime}}{\left(t^{\prime}-z^{\prime}\right) t^{\prime}} d t^{\prime}, \quad B^{\prime}=\text { const }>0 .
$$

We split the latter integral into two:

$$
\frac{1}{2 \pi i} \int_{\left|t^{\prime}\right| \leq B^{\prime}} \omega\left(t^{\prime}\right)\left(\frac{1}{t^{\prime}-z^{\prime}}-\frac{1}{t^{\prime}}\right) d t^{\prime}=\frac{1}{2 \pi i} \int_{\left|t^{\prime}\right| \leq B^{\prime}} \frac{\omega\left(t^{\prime}\right) d t^{\prime}}{t^{\prime}-z^{\prime}}-\frac{1}{2 \pi i} \int_{\left|t^{\prime}\right| \leq B^{\prime}} \frac{\omega\left(t^{\prime}\right) d t^{\prime}}{t^{\prime}} .
$$

The first part of Theorem 2.1 follows from this formula and the results on Cauchy integrals for the case of $z$ varying in a bounded domain.

Now, suppose that, for $|t| \geq$ const $>0$, we have

$$
\omega(t)=\frac{1}{t} \omega_{1}(t), \quad \omega_{1}(t) \in \operatorname{Lip} \alpha(|t| \geq \text { const }>0) .
$$

Then

$$
f(z)=\frac{1}{2 \pi i} \int_{|t| \leq \mathrm{const}} \frac{\omega(t) d t}{t-z}+\frac{1}{2 \pi i} \int_{|t| \geq \mathrm{const}} \frac{\omega(t) d t}{t-z}=f_{1}(z)+f_{2}(z) .
$$

Here $f_{1}(z)=O\left(\frac{1}{z}\right)$ and $f_{1}(z)$ is regular near $z=\infty$. In the second integral we change the variables: $t^{\prime}=\frac{1}{t}, z^{\prime}=\frac{1}{z}$. Then

$$
f_{2}(z)=\frac{1}{2 \pi i} \int_{\left|t^{\prime}\right| \leq \mathrm{const}} \frac{\omega\left(t^{\prime}\right)}{\frac{1}{t^{\prime}}-\frac{1}{z^{\prime}}} \frac{d t^{\prime}}{-t^{\prime 2}}=z^{\prime} \frac{1}{2 \pi i} \int_{\left|t^{\prime}\right| \leq \mathrm{const}} \frac{\omega_{1}\left(t^{\prime}\right) d t^{\prime}}{t^{\prime}-z}
$$

which implies

$$
f_{2}=\frac{1}{z} f_{3}(z), \quad f_{3}(z) \in \operatorname{Lip} \alpha\left(\Pi_{ \pm} \cap|z| \geq \text { const }>0\right) .
$$

\section{REFERENCES}

[1] B. Noble, Methods based on the Wiener-Hopf technique for the solution of partial differential equations, Internat. Ser. Monogr. Pure Appl. Math., vol. 7, Pergamon Press, New York, 1958. MR0102719(21:1505)

[2] R. Mittra and S. W. Lee, Analytical techniques in the theory of guided waves, Macmillan, New York, 1971.

[3] L. A. Takhtadzhyan and L. D. Faddeev, Hamiltonian methods in the theory of solitons, "Nauka", Moscow, 1986; English transl., Springer-Verlag, Berlin, 1987. MR0889051|(89m:58102) MR0905674 (89m:58103)

[4] S. Novikov, S. V. Manakov, L. E. Pitaevskiŭ, and V. E. Zakharov, Theory of solitons. The inverse scattering method, "Nauka", Moscow, 1980; English transl., Consultants Bureau [Plenum], New York, 1984. MR0573607 (81g:35112) MR0779467 (86k:35142)

[5] J. Plemelj, Riemannsche Funktionenscharen mit gegebener Monodromiegruppe, Monatsh. Math. Phys. 19 (1908), 211-245. MR 1547764

[6] N. I. Muskhelishvili, Singular integral equations. Boundary problems of function theory and their application to mathematical physics, Gostekhizdat, Moscow-Leningrad, 1946; English transl., Noordhoff Internat. Publ., Leyden, 1977. MR0020708 (8:586b) MR.0438058 (55:10978)

[7] N. P. Vekua, Systems of singular integral equations and some boundary problems, GITTL, MoscowLeningrad, 1950; English transl., P. Noordhoff, Ltd., Groningen, 1967. MR0043346 (13:247a) MR 0211220 (35:2102)

[8] S. G. Michlin and S. Prössdorf, Singuläre Integraloperatoren, Math. Lehrbücher Monogr. II. Abt. Math. Monogr., Bd. 52, Akademie-Verlag, Berlin, 1980. MR0587117 (82c:45002) 
[9] I. Ts. Gokhberg and M. G. Kreı̆n, Systems of integral equations on the half-line with kernels depending on the difference of the arguments, Uspekhi Mat. Nauk 13 (1958), no. 2(80), 3-72; English transl., Amer. Math. Soc. Transl. (2), vol. 14, Amer. Math. Soc., Providence, RI, 1960, pp. 217-287. MR0102720 (21:1506)

[10] F. D. Gakhov, Boundary value problems, Fizmatgiz, Moscow, 1958; English transl., Pergamon Press, Oxford, 1966. MR0104117 (21:2879) MR0198152 (33:6311)

[11] S. L. Sobolev, Some applications of functional analysis in mathematical physics, 3rd ed., "Nauka", Moscow, 1988; English transl., Transl. Math. Monogr., vol. 90, Amer. Math. Soc., Providence, RI, 1991. MR0986735(90m:46059) MR1125990(92e:46067)

St. Petersburg Branch, Steklov Mathematical Institute, Russian Academy of Sciences, Fontanka 27, St. Petersburg 191023, Russia

E-mail address: babich@pdmi.ras.ru

Received 8/FEB/2007

Translated by A. PLOTKIN 